\title{
Weight loss reduces head motion: Revisiting a major confound in neuroimaging
}

\author{
Frauke Beyer, ${ }^{1,2}$ | Kristin Prehn ${ }^{3,4}$ | Katharina A. Wüsten ${ }^{5,6}$ | Arno Villringer ${ }^{1,2}$ | \\ Jürgen Ordemann $^{7,8}$ | Agnes Flöel ${ }^{3,5,6,9} \quad$ A. Veronica Witte ${ }^{1,2}$
}

${ }^{1}$ Department of Neurology, Max Planck Institute for Human Cognitive and Brain Sciences, Leipzig, Germany

${ }^{2}$ Subproject A1, CRC 1052 "Obesity Mechanisms", University of Leipzig, Leipzig, Germany

${ }^{3}$ Department of Neurology \& NeuroCure Clinical Research Center, Charité University Medicine, Berlin, Germany

${ }^{4}$ Department of Psychology, Medical School Hamburg, Hamburg, Germany

${ }^{5}$ Department of Neurology, University of Greifswald, Greifswald, Germany

${ }^{6}$ German Center for Neurodegenerative Diseases, Standort Rostock/Greifswald, Greifswald, Germany

${ }^{7}$ Center for Bariatric and Metabolic Surgery, Charité University Medicine, Berlin, Germany

${ }^{8}$ Zentrum für Adipositas und Metabolische Chirurgie, Vivantes Klinikum Spandau, Berlin, Germany

${ }^{9}$ Center for Stroke Research, Charité University Medicine, Berlin, Germany

Correspondence

A. Veronica Witte, Department of Neurology, Max Planck Institute for Cognitive and Brain Sciences, Stephanstr. 1a, 04103 Leipzig, Germany.

Email: witte@cbs.mpg.de

Funding information

Deutsche Forschungsgemeinschaft, Grant/ Award Numbers: 209933838 - SFB 1052, DFG-Exc 257, FL 379-16/1, FI 379-10/1, FI 379-11/1, SFB1315 TP B03, WI 3342/3-1

\begin{abstract}
Head motion during magnetic resonance imaging (MRI) induces image artifacts that affect virtually every brain measure. In parallel, cross-sectional observations indicate a correlation of head motion with age, psychiatric disease status and obesity, raising the possibility of a systematic artifact-induced bias in neuroimaging outcomes in these conditions, due to the differences in head motion. Yet, a causal link between obesity and head motion has not been tested in an experimental design. Here, we show that a change in body mass index (BMI) (i.e., weight loss after bariatric surgery) systematically decreases head motion during MRI. In this setting, reduced imaging artifacts due to lower head motion might result in biased estimates of neural differences induced by changes in BMI. Overall, our finding urges the need to rigorously control for head motion during MRI to enable valid results of neuroimaging outcomes in populations that differ in head motion due to obesity or other conditions.
\end{abstract}

\section{KEYWORDS}

body mass index, head motion, imaging artifact, neuroimaging, obesity, resting state fMRI

\section{1 | INTRODUCTION}

Head motion is an important confounder in neuroimaging studies of brain structure and function (Power, Schlaggar, \& Petersen, 2015; Savalia et al., 2017). Micro-movements of the head, driven by spontaneous motion or respiration, strongly affect qualitative and quantitative neuroimaging outcomes, even if targeted image processing techniques are used (Parkes, Fulcher, Yücel, \& Fornito, 2018). Moreover, head motion during MRI often correlates with the predictors under study, such as age, psychiatric disease status and

This is an open access article under the terms of the Creative Commons Attribution-NonCommercial License, which permits use, distribution and reproduction in any medium, provided the original work is properly cited and is not used for commercial purposes.

(c) 2020 The Authors. Human Brain Mapping published by Wiley Periodicals, Inc. 
obesity (Hodgson et al., 2017; Makowski, Lepage, \& Evans, 2019; Torres \& Denisova, 2016). This raises the possibility of a systematic image artifact-induced bias due to differences in head motion in these conditions (Pardoe et al., 2016; Satterthwaite et al., 2012).

One of the strongest predictors of head motion is body mass index (BMI) (Beyer et al., 2017; Ekhtiari, Kuplicki, Yeh, \& Paulus, 2019; Hodgson et al., 2017). It seems likely that physiological differences associated with higher weight, for example, increased respiratory rate and amplitude, or spontaneous motion due to uncomfortable positioning in the magnet bore may induce this effect (Littleton, 2012). Yet, within-subject analysis have indicated that differences in head motion may be also driven by a neurobiological trait which shares genetic variance with BMI (Hodgson et al., 2017; Zeng et al., 2014). Along these lines, impulsivity, for example, more rash action tendencies, might explain a proportion of the shared variance between head motion and obesity (Couvy-Duchesne et al., 2016; Kong et al., 2014).

Until now, mainly cross-sectional studies report on the association of BMI and head motion and little is known about how BMI changes may affect head micro-movements. In this pre-registered analysis (https://osf.io/epsxt), we therefore aimed to test whether a radical weight-loss intervention (bariatric surgery) compared to a control group induces consistent changes of head motion during magnetic resonance imaging (MRI) in obese individuals. In addition, we aimed to estimate the confounding effects of a potential change in head motion after bariatric surgery on structural brain measures, according to previous literature (Madan, 2018; Reuter et al., 2015; Savalia et al., 2017).

\section{2 | METHODS}

To test whether a decrease in BMI would reduce head micromovements during MRI, we investigated the effects of bariatric surgery on head motion in patients from the Center for Bariatric and Metabolic Surgery at Charité University Medicine Berlin. The local Ethics Committee of the Charité University Medicine Berlin approved the study protocol and the study was carried out in accordance with the principles of the Declaration of Helsinki. All subjects provided written informed consent and received a small reimbursement for their participation.

This analysis was preregistered (https://osf.io/epsxt). At the date of the preregistration, the acquisition of the data had been finished and the authors had seen summary statistics of BMI for both time points and groups, but the main outcomes (mean and maximal framewise displacement (FD) had not been calculated.

From the total study sample, we included all patients who received a MRI (for more details see (Prehn et al., 2020)). We compared 33 obese participants who underwent bariatric surgery (scanned at baseline $n=21$, no baseline BMI information for one participant, $7 \mathrm{~m} / 26 \mathrm{f}$, aged $43.0 \pm 11.9$ years, BMI: $46.4 \pm 5.9 \mathrm{~kg} / \mathrm{m}^{2}$ (mean $\pm S D)$ ) to 17 obese participants in a waiting list-control (scanned at baseline $n=16,6 \mathrm{~m} / 11 \mathrm{f}$, aged: $47.5 \pm 10.9$ years, BMI 43.7 $\pm 5.2 \mathrm{~kg} / \mathrm{m}^{2}$ ). Patients in the control group did not differ significantly from the intervention group regarding baseline characteristics. They had also been recommended to undergo bariatric surgery, but could not be scheduled for this procedure as they had to wait for the approval by their health insurances.

Resting state functional MRI (rsfMRI) $\left(T_{2}{ }^{*}\right.$-weighted EPI sequence, 150 volumes, 34 slices, repetition time $2000 \mathrm{~ms}$, echo time $=30 \mathrm{~ms}$, flip angle $=90^{\circ}$, voxel size $=3.0 \times 3.0 \times 4.0 \mathrm{~mm}$ ) was acquired at three time points maximum (baseline, 6 and 12 months after surgery or waiting period) on a 3 Tesla Siemens Trio MRI. 11/10 participants from intervention/control group completed all three assessments and all participants were included into the linear mixed model analysis. Mean and maximal FD were calculated according to (Power, Barnes, Snyder, Schlaggar, \& Petersen, 2012). We deviated from the preregistration by log-transforming mean and maximal FD values prior to the analysis.

To test the hypothesis of a significant interaction of time point with group on mean FD, we set up a linear mixed model with group, time point and their interaction as fixed effects and subject as random effect. We used the function Imer of the R-package Ime4 in R version 3.6.1 (R Core Team, 2019) (Full model H1.1 = Imer(mean FD $\sim$ group $\times$ time point $+(1 \mid$ subj $))$. We checked the assumption of normally distributed residuals by visually inspecting the qq-plot and saw no obvious deviations. We reported regression coefficients and $R^{2}$ of the fixed effects of this model, calculated with the function $r$. squaredGLMM from the R-package MuMin. To test the significance of the interaction effect between time point and group, we compared the full model including the interaction of group and time point to a null model including only main effects (Null model H1.0 = Imer(mean FD group + time $+(1$ lsubj))). Here, we deviated from the wrong specification in the preregistration by including the main effect of group, which is necessary to restrict the test to the interaction. We reported $X^{2}$ and $p$-values of the likelihood ratio test $(R$ function ANOVA) of the interaction, as well as $T$ and $p$-values of fixed effect estimates calculated with the R-package ImerTest. Within-subject error for plotting was calculated using freely available R-scripts (http://www.cookbook-r.com/Graphs/Plotting_means_and_error_ bars_(ggplot2)/\#Helper\%2Ofunctions) based on (Morey, 2008).

To more comprehensively describe the relation of head motion and $\mathrm{BMI}$ we tested the hypothesis that within-subject BMI change did not predict within-subject head motion change. If we failed to reject this hypothesis, we would not be able to claim that BMI was a strong determinant of head motion. In this preregistered secondary analysis, we calculated within- and between-subject BMI variability as the average and difference of BMI across time points. For example, if patients had all three time points, this resulted in one value for the average $\mathrm{BMI}$ and three values for the difference between the time point $\mathrm{BMI}$ and the mean. Participants with only one measurement were assigned to zero within-subject variability. We then used a likelihood ratio test to compare the full model including both within- and between-subject BMI $\left(\mathrm{H} 2.1=\operatorname{Imer}\left(\right.\right.$ Mean FD $\sim \mathrm{BMI}_{\text {between }}+\mathrm{BMI}_{\text {within }}+(1 \mid$ subj $\left.\left.)\right)\right)$ to a reduced model (Null model H2.0 = Imer(Mean $\mathrm{FD} \sim \mathrm{BMI}_{\text {between }}+(1 \mid$ subj))), which included only between-subject BMI. For this analysis, we reported $\mathrm{R}^{2}$ of the fixed effects of the full model and $\mathrm{X}^{2}$ and $\mathrm{p}$-value of the likelihood ratio test. 
TABLE 1 Results for the fixed effects from the linear mixed model ( $\mathrm{H} 1.1)$ predicting logarithmized mean FD (log mean FD $\sim$ group $\times$ time point). Time point has the levels baseline (reference level), 6 and 12 month follow-up (FU). Condition has the levels control group (reference level) and intervention group (IG). Shown are the $\beta$ estimates, standard errors (SE), T- and $p$ values for the main effects of time point (6/12 month FU, compared to baseline), the main effect of condition (IG, compared to control group) and the interaction of time point and condition (6 month FU:IG and 12 month FU:IG, difference in change from baseline to 6/12 month FU in IG compared to control group)

\begin{tabular}{|lllll|}
\hline Fixed effects & $\boldsymbol{\beta}$ estimate & $\boldsymbol{\beta}$ SE & $\boldsymbol{T}$ value & $\boldsymbol{p}$ Value \\
\hline (Intercept) & 0.54 & 0.07 & -8.2 & $<.001$ \\
\hline 6 month FU & 0.06 & 0.05 & 1.2 & .22 \\
\hline 12 month FU & 0.0004 & 0.05 & 0.009 & .99 \\
\hline IG & -0.063 & 0.08 & -0.8 & .45 \\
\hline 6 month FU:IG & -0.21 & 0.06 & -3.4 & .0014 \\
\hline 12 month FU:IG & -0.17 & 0.07 & -2.4 & .019 \\
\hline
\end{tabular}

We also repeated the above analyses with maximal FD as outcome measure $(H 3.1=\operatorname{Imer}(\operatorname{Max} F D \sim$ group $\times$ time point $+(1 \mid$ subj $))$, H3.0 = Imer(Max FD group + time point + (1|subj)), H4.1 = Imer(Max $\mathrm{FD} \sim \mathrm{BMI}_{\text {between }}+\mathrm{BMI}_{\text {within }}+(1 \mid$ subj) $)$, Null model H4.0 = Imer(Mean $\mathrm{FD} \sim \mathrm{BMI}_{\text {between }}+(1 \mid$ subj $\left.\left.)\right)\right)$.

All code for this analysis is publicly available under https://github. com/fBeyer89/ADI_preproc/tree/master/Project1_headmotion. The data that support the findings of this study are available from the corresponding author upon request.

\section{3 | RESULTS}

We compared head motion during rsfMRI of 33 obese participants that underwent bariatric surgery to 17 obese participants in a waiting list-control group at baseline, 6 and 12 months after surgery/waiting period. Bariatric surgery compared to control led to a systematic reduction of head motion, measured using mean FD of 150 individual brain volumes acquired with a 6 min scan (linear mixed models H.1.1 and H1.0 compared with a likelihood-ratio test, $X^{2}=10.8, d f=2$, $p=.0043, R^{2}$ of fixed effects: .15 , based on 107 observations from 50 participants).

Average head motion of control participants did not change, while participants in the intervention group had lower mean FD at 6 and 12 month follow-up (see Table 1 and Figure 1).

Moreover, the magnitude of weight loss, measured as change in $\mathrm{BMI}$, predicted the decrease in mean FD (linear mixed models H2.1 and $\mathrm{H} 2.0$ compared with a likelihood-ratio test, $X^{2}=20.6, d f=1$, $p<.001, R^{2}$ of fixed effects: .053, based on 107 observations from 50 participants, see Figure 2a,b).

There was no time point by group interaction for maximal FD (linear mixed models H.3.1 and H3.0 compared with a likelihood-ratio test, $X^{2}=2.2, d f=2, p=.33, R^{2}$ of fixed effects: .11 , based on

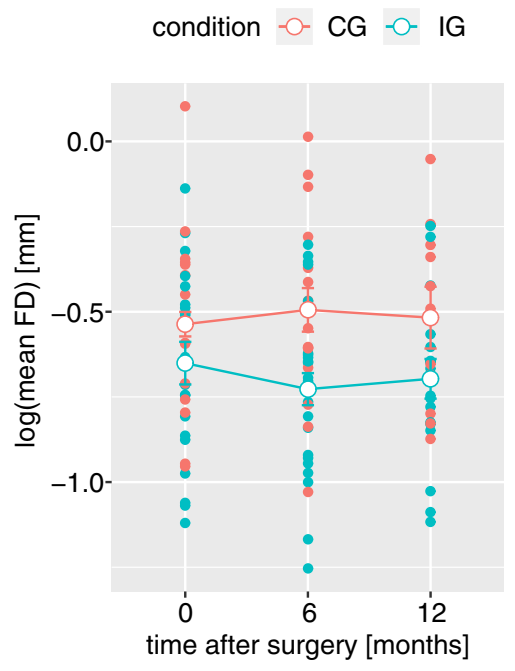

FIGURE 1 Head motion during resting state fMRI, measured as log-normalized mean framewise displacement (mean FD), decreases in the intervention (IG) compared to the control group (CG), shown in blue/red, respectively. Open dots represent group and time point averages, error bars represent within-subject errors

107 observations from 50 participants). Yet, similar to the findings for mean FD, the magnitude of weight loss did predict differences in maximal FD (linear mixed models $\mathrm{H} 4.1$ and $\mathrm{H} 4.0$ compared with a likelihood-ratio test, $X^{2}=6, d f=1, p=.014, R^{2}$ of fixed effects: .035 , based on 107 observations from 50 participants).

To estimate the impact of head motion changes on structural imaging outcomes, we assumed an average total gray matter volume of $\sim 600 \mathrm{~cm}^{3}$ (based on own data) and $24 \mathrm{~cm}^{3}$ gray matter loss per $0.1 \mathrm{~mm}$ of mean FD increase based on results from Alexander-Bloch et al. (Alexander-Bloch et al., 2016). Thus, an estimated decrease in mean FD from baseline to follow up in the intervention group of $0.12 \mathrm{~mm}$, as shown in the current analysis, would translate into a false increase in total gray matter volume of $28.8 \mathrm{~cm}^{3}$ (or about 5\%) after bariatric surgery, due to the decrease in head motion alone.

\section{4 | DISCUSSION}

In this preregistered analysis, we showed that a radical change in physiological parameters, in this case body weight loss induced by bariatric surgery, reduced head micro-movements during MRI. The magnitude of changes in BMI further predicted the magnitude of reduction in head motion. This indicates that head motion strongly depends on body physiology and that BMI differences may result in biased estimates of brain structure and function. Our result highlights the need of rigorous attempts to adjust for and reduce head motion during neuroimaging studies in obesity, as well as in other conditions that may be systematically related to head motion.

Our findings are in line with previous observations in several large imaging cohorts in which BMI accounted for $8-40 \%$ of the variance in head motion, making it one of the most important predictors of head 
(a)

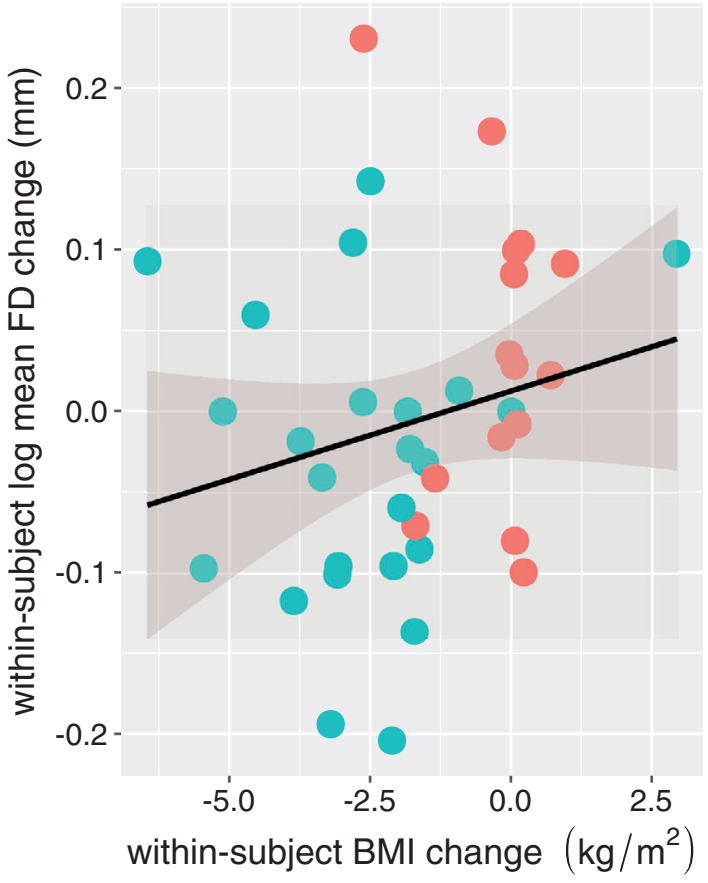

(b)

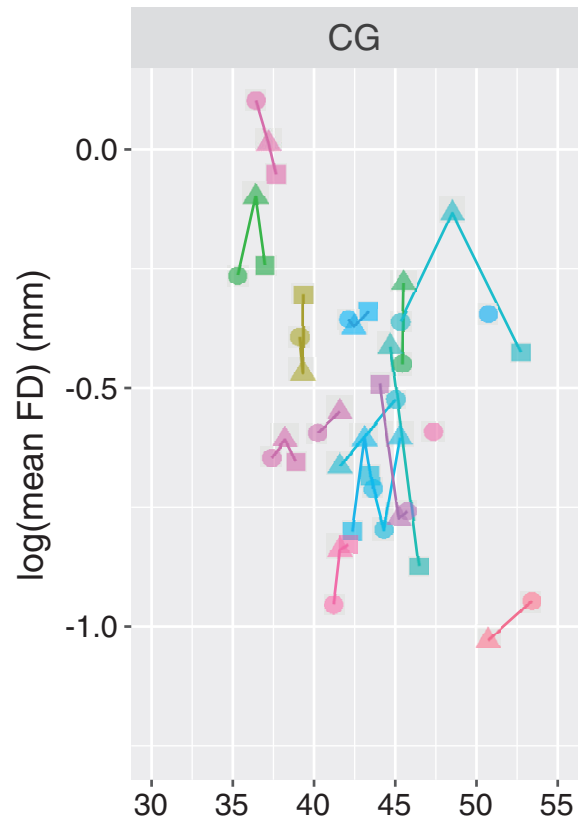

BMI $\left(\mathrm{kg} / \mathrm{m}^{2}\right)$

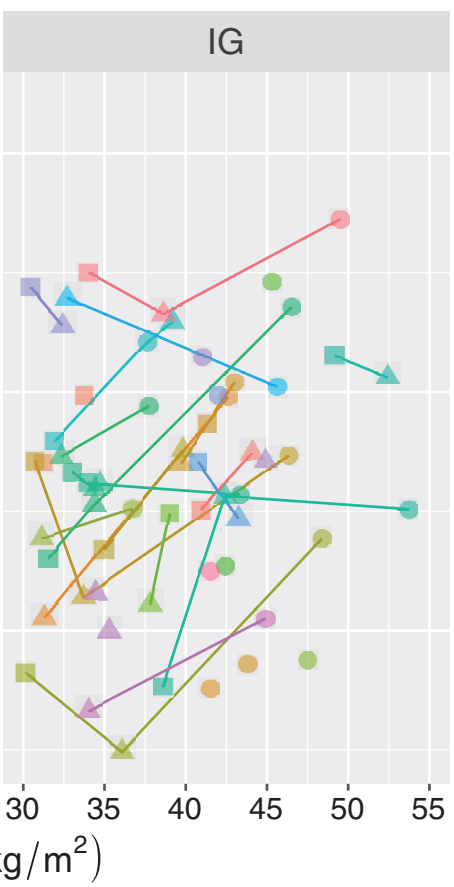

FIGURE 2 (a) The within-subject change in BMI ( $x$-axis) from baseline to 6 month follow up predicts the change in mean FD ( $y$-axis). Color represents the condition (red: control group (CG), blue: intervention group (IG)). The line shows the regression curve with $95 \%$ confidence intervals. (b) Detailed BMI and mean FD trajectories for CG (left) and IG (right). Each color represents one participant. Time after surgery is symbolized by a circle ( 0 months), triangle ( 6 months) and square (12 months). BMI, body mass index; FD, framewise displacement

motion (Ekhtiari et al., 2019; Hodgson et al., 2017). While our estimate of a weight loss-induced bias in neuroimaging outcomes (i.e., $5 \%$ increase in total gray matter volume after bariatric surgery) relied on between-subject estimates of the effect of head motion (AlexanderBloch et al., 2016) and therefore has to be interpreted with caution, this finding still stresses the importance to control for head motion differences in future MRI analyses. Different techniques, such as multi-echo sequences (Power et al., 2018), fixation through head molds (Power et al., 2019) and tactile feedback during scanning (Krause et al., 2019) have been proposed to considerably reduce head motion a priori-which is probably the best way to handle this important confound for practically all imaging outcomes (Baum et al., 2018; Beyer et al., 2017; Madan, 2018; Reuter et al., 2015).

Little is known about the mechanisms that may underlie the causal link between body weight and head motion. Possibly, obesityrelated alterations in the respiratory system lead to increased real and apparent head motion in MRI scans (Littleton, 2012). Yet, the association of $\mathrm{BMI}$ and head motion was not mediated by respiration rate in previous studies and has been reported for datasets with both short and long imaging repetition times (Beyer et al., 2017; Ekhtiari et al., 2019; Hodgson et al., 2017). More speculatively, alterations in the brain's dopaminergic system could represent a link between BMI and head motion. Evidence suggests that BMI-related differences in dopamine receptor availability might underlie observed differences in dopamine-related functions such as reward sensitivity (Tomasi \&
Volkow, 2013). Given that dopaminergic signaling is crucial for motor inhibition and control (Cools \& D'Esposito, 2011; Robertson et al., 2015), alterations in the dopaminergic system might also influence spontaneous head motion.

A limitation of this study is that we did not assess other predictors of head motion such as impulsivity, respiration or dopaminergic signaling. We therefore cannot exclude that these measures mediate the observed effect. Yet, our main conclusion that physiological changes induce changes in head motion, and its implications for future studies, remain valid regardless of the exact mechanisms or other influencing factors. Future studies are encouraged to further investigate the complex interplay of physiological and psychological factors in bariatric surgery (see for example https://osf.io/adnqc).

Taken together, radical weight loss induced by bariatric surgery reduces head motion in a cohort of obese individuals, indicating that the physiological state strongly determines higher head motion in obesity. This urges adequate a-priori control of head motion in neuroimaging studies of obesity and other conditions with systematically increased head motion to eliminate its confounding effects on measures of brain structure and function.

\section{ACKNOWLEDGMENTS}

We thank T. Profitlich, S. Heßler, I. Rangus, C. Reschke, L. Kaiser, A. Winkler, L. Kerti for help in data acquisition. This work was supported by the German Research Foundation, contract grant 
numbers WI 3342/3-1, FI 379-10/1, FI 379-11/1, FL 379-16/1, DFGExc 257, SFB1315 TP B03 and CRC 1052 "Obesity mechanisms" Project $A 1$.

\section{DATA AVAILABILITY STATEMENT}

The data that support the findings of this study are available from the corresponding author upon request.

\section{ORCID}

Frauke Beyer (D) https://orcid.org/0000-0001-5401-852X

\section{REFERENCES}

Alexander-Bloch, A., Clasen, L., Stockman, M., Ronan, L., Lalonde, F., Giedd, J., \& Raznahan, A. (2016). Subtle in-scanner motion biases automated measurement of brain anatomy from in vivo MRI. Human Brain Mapping, 37, 2385-2397.

Baum, G. L., Roalf, D. R., Cook, P. A., Ciric, R., Rosen, A. F. G., Xia, C., ... Tunç, B. (2018). The impact of in-scanner head motion on structural connectivity derived from diffusion MRI. Neurolmage, 173, 275-286.

Beyer, F., Kharabian Masouleh, S., Huntenburg, J. M., Lampe, L., Luck, T., Riedel-Heller, S. G., ... Witte, A. V. (2017). Higher body mass index is associated with reduced posterior default mode connectivity in older adults. Human Brain Mapping, 38, 3502-3515.

Cools, R., \& D'Esposito, M. (2011). Inverted-U-shaped dopamine actions on human working memory and cognitive control. Biological Psychiatry, 69, e113-e125.

Couvy-Duchesne, B., Ebejer, J. L., Gillespie, N. A., Duffy, D. L., Hickie, I. B., Thompson, P. M., ... Wright, M. J. (2016). Head motion and inattention/hyperactivity share common genetic influences: Implications for fMRI studies of ADHD. PLoS One, 11, e0146271.

Ekhtiari, H., Kuplicki, R., Yeh, H.-w., \& Paulus, M. P. (2019). Physical characteristics not psychological state or trait characteristics predict motion during resting state fMRI. Scientific Reports, 9, 419.

Hodgson, K., Poldrack, R. A., Curran, J. E., Knowles, E. E., Mathias, S., Göring, H. H. H., ... Almasy, L. (2017). Shared genetic factors influence head motion during MRI and body mass index. Cerebral Cortex, 27(12), $5539-5546$.

Kong, X. Z., Zhen, Z., Li, X., Lu, H. H., Wang, R., Liu, L., ... Liu, J. (2014). Individual differences in impulsivity predict head motion during magnetic resonance imaging. PLoS One, 9, e104989.

Krause, F., Benjamins, C., Eck, J., Lührs, M., van Hoof, R., \& Goebel, R. (2019). Active head motion reduction in magnetic resonance imaging using tactile feedback. Human Brain Mapping, 40, 4026-4037.

Littleton, S. W. (2012). Impact of obesity on respiratory function. Respirology, 17, 43-49.

Madan, C. R. (2018). Age differences in head motion and estimates of cortical morphology. PeerJ, 6, e5176.

Makowski, C., Lepage, M., \& Evans, A. C. (2019). Head motion: The dirty little secret of neuroimaging in psychiatry. Journal of Psychiatry \& Neuroscience, 44, 62-68.

Morey, R. D. (2008). Confidence intervals from normalized data: A correction to Cousineau (2005), 4, 61-64.

Pardoe, H. R., Hiess, R. K., Kuzniecky, R. (2016). Motion and morphometry in clinical and nonclinical populations. Neuroimage, 135, 177-185.
Parkes, L., Fulcher, B., Yücel, M., \& Fornito, A. (2018). An evaluation of the efficacy, reliability, and sensitivity of motion correction strategies for resting-state functional MRI. Neurolmage, 171, 415-436.

Power, J., Schlaggar, B., \& Petersen, S. (2015). Recent progress and outstanding issues in motion correction in resting state fMRI. Neurolmage, 105, 536-551.

Power, J. D., Barnes, K. A., Snyder, A. Z., Schlaggar, B. L., \& Petersen, S. E. (2012). Spurious but systematic correlations in functional connectivity MRI networks arise from subject motion. Neurolmage, 59, 2142-2154.

Power, J. D., Plitt, M., Gotts, S. J., Kundu, P., Voon, V., Bandettini, P. A., \& Martin, A. (2018). Ridding fMRI data of motion-related influences: Removal of signals with distinct spatial and physical bases in multiecho data. Proceedings of the National Academy of Sciences of the United States of America, 115, E2105-E2114.

Power, J. D., Silver, B. M., Silverman, M. R., Ajodan, E. L., Bos, D. J., \& Jones, R. M. (2019). Customized head molds reduce motion during resting state fMRI scans. Neurolmage, 189, 141-149.

Prehn, K., Profitlich, T., Rangus, I., Heßler, S., Witte, A. V., Grittner, U., ... Flöel, A. (2020). Bariatric surgery and brain health-A longitudinal observational study investigating the effect of surgery on cognitive function and Gray matter volume. Nutrients, 12, 127.

R Core Team. (2019). R: A Language and Environment for Statistical Computing.

Reuter, M., Tisdall, M. D., Qureshi, A., Buckner, R. L., van der Kouwe, A. J. W., \& Fischl, B. (2015). Head motion during MRI acquisition reduces gray matter volume and thickness estimates. Neurolmage, 107, 107-115.

Robertson, C. L., Ishibashi, K., Mandelkern, M. A., Brown, A. K., Ghahremani, D. G., Sabb, F., ... London, E. D. (2015). Striatal D1- and D2-type dopamine receptors are linked to motor response inhibition in human subjects. The Journal of Neuroscience, 35, 5990-5997.

Satterthwaite, T. D., Wolf, D. H., Loughead, J., Ruparel, K., Elliott, M. A., Hakonarson, H., ... C., Gur, R. E. (2012). Impact of in-scanner head motion on multiple measures of functional connectivity: relevance for studies of neuro development in youth. Neuroimage, 60, 623-632.

Savalia, N. K., Agres, P. F., Chan, M. Y., Feczko, E. J., Kennedy, K. M., \& Wig, G. S. (2017). Motion-related artifacts in structural brain images revealed with independent estimates of in-scanner head motion. Human Brain Mapping, 38, 472-492.

Tomasi, D., \& Volkow, N. D. (2013). Striatocortical pathway dysfunction in addiction and obesity: Differences and similarities. Critical Reviews in Biochemistry and Molecular Biology, 48, 1-19.

Torres, E. B., \& Denisova, K. (2016). Motor noise is rich signal in autism research and pharmacological treatments. Scientific Reports, 6, 37422.

Zeng, L. L., Wang, D., Fox, M. D., Sabuncu, M., Hu, D., Ge, M., ... Liu, H. (2014). Neurobiological basis of head motion in brain imaging. Proceedings of the National Academy of Sciences of the United States of America, 111, 6058-6062.

How to cite this article: Beyer F, Prehn K, Wüsten KA, et al. Weight loss reduces head motion: Revisiting a major confound in neuroimaging. Hum Brain Mapp. 2020;1-5. https://doi.org/ 10.1002/hbm.24959 